\title{
Application of Conditional Averaging to Time Delay Estimation of Random Signals
}

\author{
Robert Hanus, Adam Kowalczyk, Anna Szlachta, Rafał Chorzępa \\ Rzeszów University of Technology, Faculty of Electrical and Computer Engineering, 12 Powstańców Warszawy Ave., \\ 35-959 Rzeszów, Poland, rohan@prz.edu.pl
}

\begin{abstract}
The article presents the possibilities of using the function of conditional average value of a delayed signal (CAV) and the function of conditional average value of a delayed signal absolute value (CAAV) to determine the time delay estimation (TDE) of random signals. For discrete CAV and CAAV estimators, the standard uncertainties of the estimation of function values at extreme points and the standard uncertainties of the TDE were given and compared with the corresponding uncertainties for the direct discrete cross-correlation function (CCF) estimator. It was found that the standard uncertainty of TDE for CAV is lower than for CCF independent of signal-to-noise ratio (SNR) for parameter values of $\alpha \geq 2$ and $M / N \geq 0.25$ (where: $\alpha$ - relative threshold value, $M / N$ - quotient of number of averaging and number of samples). The standard uncertainty of TDE for CAAV will be lower than for CCF for SNR values greater than 0.35 (for $N / M=1$ ).
\end{abstract}

Keywords: Time delay estimation, random signals, conditional averaging, cross-correlation.

\section{INTRODUCTION}

Time delay estimation (TDE) is a common problem in signal processing. This problem is important, inter alia, in radar technology, radioastronomy, medicine, localization of disturbance pathways, and non-contact measurements of two-phase flows. For the determination of time delay of stochastic signals obtained from two or more sensors, statistical methods are commonly used. This issue is widely presented in the literature, among others [1]-[10], where a number of methods based on analysis of time and frequency signals are described. The range of applications of particular methods depends on the characteristics of the measurement signals (stationarity, probability distribution, correlation), signal-to-noise ratio (SNR), and analysis parameters. Among the classical methods used for Gaussian stationary signals, the most common are the cross-correlation function (CCF) in the time domain and phase of cross-spectral density in the frequency domain [1], [8], [10]-[15]. For the above types of signals under certain conditions, differential and combined methods [4]-[6], the Hilbert Transform-based correlation method [16]-[17], and the relatively little-known methods based on conditional averaging [18]-[20] can be used.

This paper presents the applicability of the conditional average value of the delayed signal (CAV) and the conditional average value of the delayed signal absolute value (CAAV) to the TDE in relation to the CCF. For the selected signal models, the range is determined for the SNR values for which the methods under consideration have lower standard uncertainties than the CCF method with the specified analysis parameters.

The paper is organized as follows. Section 2 gives the mathematical models of measurement random signals. The basics of the TDE using the classical cross-correlation method are reviewed in section 3 . Section 4 presents the application of conditional averaging methods CAV and CAAV to TDE and the analysis of measurement uncertainties for these methods in comparison with the CCF. The last section contains the summary of the presented study and final conclusions. This article is an extended and improved version of a previous conference publication [20].

\section{Models OF SIGNALS}

Measurement of the time delay of random signals during their propagation in physical systems involves active and passive experiments. In the active experiment, external random extortion is used. In the passive experiment, natural conditions in the object of measurement are used to generate measurement signals. The full measurement model should take into account inaccuracies in terms of input and output signals, resulting from difficult experimental conditions, and the impact of disturbance. Simplification of the random signal time delay measurement model may lead to a reduction in accuracy of the TDE. 
In time delay estimation, the models of stochastic signals $x(t)$ and $z(t)$ obtained under stationary conditions with two sensors in a passive experiment can be presented as [1]:

$$
\begin{gathered}
x(t)=s(t)+n_{1}(t) \\
z(t)=c\left[s\left(t-\tau_{0}\right)\right]+n_{2}(t)=y(t)+n_{2}(t)
\end{gathered}
$$

where: $s(t)$ and $z(t)$ - observed input and output stationary random signals; $c$ - constant factor (usually $c=1$ ); $\tau_{0}-$ transmission time delay; $n_{1}(t), n_{2}(t)$ - stationary signals, uncorrelated with the signal $s(t)$ and with each other, which may in general contain constant, periodic and random components. This paper assumes that the signals $n_{1}(t)$ and $n_{2}(t)$ are stationary Gaussian white noises $\mathrm{N}\left(0, \sigma_{n 1}\right), \mathrm{N}\left(0, \sigma_{n 2}\right)$, uncorrelated with the signal $s(\mathrm{t})$ and mutually with each other.

With the above assumptions for signal models (1) and (2), the following relationships are true:

$$
\begin{gathered}
\sigma_{x}^{2}=R_{x x}(0)=\sigma_{s}^{2}+\sigma_{n 1}^{2} \\
\sigma_{z}^{2}=R_{z z}(0)=c^{2} \sigma_{s}^{2}+\sigma_{n 2}^{2} \\
R_{s z}(\tau)=c R_{s s}\left(\tau-\tau_{0}\right)+R_{n 1 n 2}\left(\tau-\tau_{0}\right)
\end{gathered}
$$

Where: $\sigma_{s}, \sigma_{x}, \sigma_{z}, \sigma_{n 1}$, and $\sigma_{n 2}$ - standard deviations of signals $s(t), x(t), z(t), n_{1}(t)$, and $n_{2}(t)$, respectively; $R_{x x}(\tau)$, $R_{z z}(\tau)$, and $R_{s s}(\tau)$ - autocorrelation functions of signals $x(t)$, $z(t)$, and $s(t)$, respectively; $\tau$ - time delay; $R_{S z}(\tau)$ - CCF of signals $s(t)$ and $z(t) ; R_{n 1 n 2}(\tau)$ - CCF of signals $n_{1}(t)$ and $n_{2}(t)$.

In the active experiment for determining the transmission time delay, the conditions of action of the test input signal are specified and controlled (no disturbances). Conversely, output noise can lead to the action of only a random component $n(t)$ with the distribution $\mathrm{N}\left(0, \sigma_{n}\right)$. With these assumptions, the models of stochastic signals received from the sensors are represented by the following formulae:

$$
\begin{gathered}
x(t)=s(t) \\
z(t)=c \cdot x\left(t-\tau_{0}\right)+n(t)=y(t)+n(t)
\end{gathered}
$$

which constitute a simplification of models (1) and (2) with the assumptions $n_{1}(t)=0$ and $n_{2}(t)=n(t)$.

Since the signals $x(t)$ and $n(t)$ are not correlated, for the delayed signal (7) we have the following equations:

$$
\begin{gathered}
\sigma_{z}=\sqrt{\left(c \sigma_{x}\right)^{2}+\sigma_{n}^{2}} \\
R_{x z}(\tau)=c R_{s s}\left(\tau-\tau_{0}\right)
\end{gathered}
$$

where $R_{x z}(\tau)$ - the cross-correlation function of signals $x(t)$ and $z(t)$.
This paper assumes that the signal $s(t)$ is low-pass white noise with bandwidth $B$. The autocorrelation function of this signal in equations (5) and (9) can be represented by:

$$
R_{s S}(\tau)=K B\left(\frac{\sin 2 \pi B \tau}{2 \pi B \tau}\right)
$$

or another modified exponential model [21].

Experimental research usually uses normal or quasinormal probability distributions of the processed signals.

\section{CoRrELATION PRINCIPLE OF TDE}

Most of the descriptions in the literature on the crosscorrelation method of determining the transmission time delay use dependencies (1) and (2) or (6) and (7). The CCF achieves the maximum value for $\tau=\tau_{0}$, so the transmission time delay can be defined as the main argument of this function (Fig.1.):

$$
\tau_{0}=\arg \left\{\max R_{x z}(\tau)\right\}=\arg \left\{R_{x z}\left(\tau_{0}\right)\right\}
$$

a)

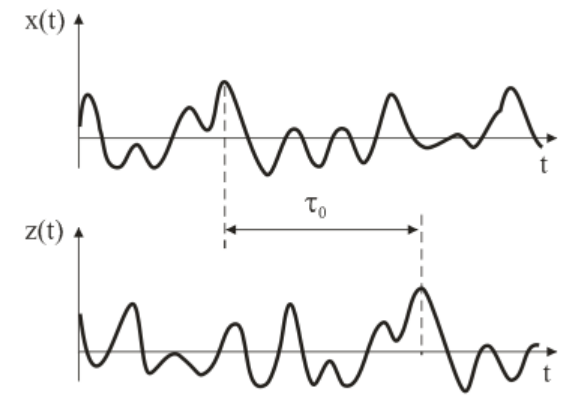

b)

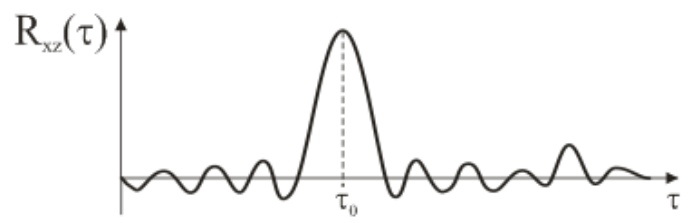

Fig.1. The concept of determining the transmission time delay $\tau_{0}$ from the cross-correlation function: a) the waveforms $x(t)$ and $z(t)$; b) the CCF $R_{x z}(\tau)$.

The normalized CCF (9) is equal to:

$$
\rho_{x z}(\tau)=\frac{R_{x z}(\tau)}{\sqrt{R_{x x}(0) R_{z z}(0)}}=\frac{c R_{S S}\left(\tau-\tau_{0}\right)}{\sigma_{x} \sigma_{z}}
$$

and after substitution $\tau=\tau_{0}$ :

$$
\rho_{X z}\left(\tau_{0}\right)=\frac{c R_{S S}(0)}{\sigma_{X} \sigma_{z}}=\frac{c \sigma_{S}^{2}}{\sigma_{X} \sigma_{z}}
$$

Assuming the same disruption at the input and output $\left(\sigma_{n 1}=\sigma_{n 2}=\sigma_{n}\right)$ for signal models (1) and (2), after substituting (3) and (4) to the equation (13), we obtain [22]: 


$$
\rho_{X Z}\left(\tau_{0}\right)=\left[1+\frac{1}{S N R}\left(1+\frac{1}{c^{2}}\right)+\frac{1}{c^{2} S N R^{2}}\right]^{-1 / 2}
$$

where

$$
\operatorname{SNR}=\left(\frac{\sigma_{s}}{\sigma_{n}}\right)^{2}
$$

Similarly, for signal models (6) and (7) we have:

$$
\rho_{x z}\left(\tau_{0}\right)=\left[1+\frac{1}{c^{2} S N R}\right]^{-1 / 2}
$$

In the absence of disruption in both cases $\rho_{x z}\left(\tau_{0}\right)=1$.

The plots of the relations (15) and (16), referred to hereafter as model I and model II, respectively, are shown in Fig.2. Particularly equations (15) and (16) and their graphs can be very useful in practice. Based on them, for obtained experimental values of normalized CCF $\rho_{x z}(\tau)$ and assumed signal models, the SNR value can be estimated at an unknown value of $n(t)$.

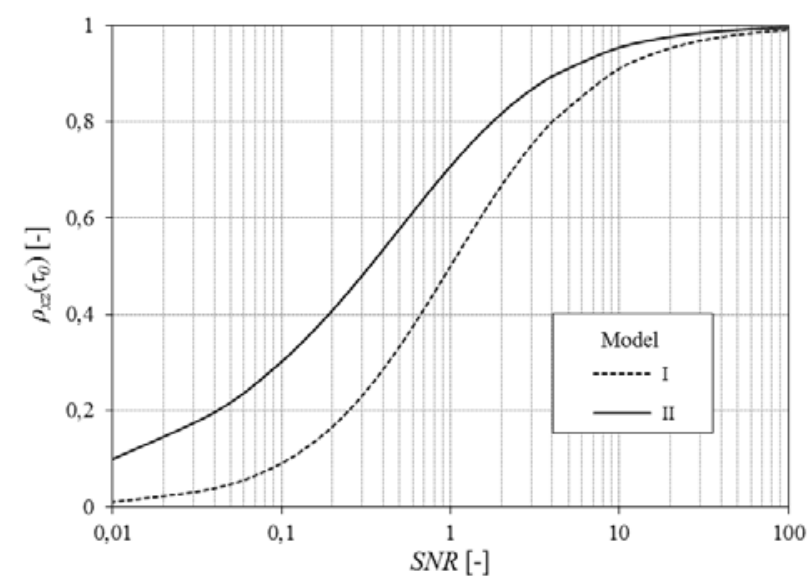

Fig.2. Plot of the relation (14) (Model I) and (16) (Model II) for $c=1$.

For further analysis, the equation (16) will be used as the most commonly used signal model in practice. If the waveforms $x(t)$ and $z(t)$ are of length $T_{\text {total }}$, the variance of the CCF estimator for $\tau=\tau_{0}$ is given by [1]:

$$
\sigma^{2}\left[\hat{R}_{x z}\left(\tau_{0}\right)\right] \approx \frac{1}{2 B T_{\text {total }}}\left[R_{x x}(0) R_{z z}(0)+R_{x z}^{2}\left(\tau_{0}\right)\right]
$$

Dependency (17) is appropriate for large values of $T_{\text {total }}$ ( $T_{\text {total }} \geq 10|\tau|$ and $B T_{\text {total }} \geq 5$ ).

For digital analysis methods, we can take $2 B T_{\text {total }}=N_{\text {total }}$ [15] where $N_{\text {total }}=\operatorname{int}\left(T_{\text {total }} / \Delta t\right)$, int - integer, and $\Delta t$ is a properly chosen sampling period. Transforming (17) we obtain:

$$
\sigma^{2}\left[\hat{R}_{x z}\left(\tau_{0}\right)\right] \approx \frac{\sigma_{x}^{2} \sigma_{z}^{2}}{N_{\text {total }}}\left[1+\rho_{x z}^{-2}\left(\tau_{0}\right)\right]
$$

When estimating CCF using pairs of uncorrelated samples divided into $N$-cycles, on the basis of (18) taking into account (16) we obtain the relative standard uncertainty of the function value:

$$
u_{\text {rel }}\left[\hat{R}_{x z}\left(\tau_{0}\right)\right]=\frac{\sigma\left[\hat{R}_{x z}\left(\tau_{0}\right)\right]}{R_{x z}\left(\tau_{0}\right)}=\frac{1}{\sqrt{N}} \sqrt{2+\frac{1}{c^{2} S N R}}
$$

A discrete CCF estimator can be expressed as:

$$
\hat{R}_{x z}(l)=\frac{1}{N-l} \sum_{n=1}^{N-l} x(n) z(n+l)
$$

where: $l=\tau / \Delta t, n=t / \Delta t$.

For a large number of samples, $N$ is used in the denominator of dependency (20) instead of $N-l$.

The standard uncertainty of transmission time delay $\tau_{0}$, determined from CCF, is given by [20]:

$$
u\left[\tau_{0}\right]_{C C F}=\frac{1}{\pi B} \sqrt[4]{\frac{3}{4}}\left\{\left(u_{r e l}\left[\hat{R}_{x z}\left(\tau_{0}\right)\right]\right)^{1 / 2}\right\}^{1 / 2}
$$

For signal models (6) and (7), using the equation (19) in (21) we obtain:

$$
u\left[\tau_{0}\right]_{C C F}=\frac{1}{\pi B} \sqrt[4]{\frac{3}{4}}\left\{\frac{1}{\sqrt{N}}\left[2+\frac{1}{c^{2} S N R}\right]^{1 / 2}\right\}^{1 / 2}
$$

\section{APPLICATION OF CONDITIONAL AVERAGING TO TDE OF THE RANDOM SIGNALS}

The transmission time delay can be determined with the use of conditional expected values: delayed signal $z(t)$ and delayed signal absolute value $|z(t)|$. Sections 4.1 and 4.2 illustrate the concept of particular methods and their standard uncertainties with respect to the cross-correlation method for signal models (6) and (7).

\subsection{Conditional average value of the delayed signal}

The conditional expected value of the delayed signal $z(t)$ (7) for the condition $x(t)=x_{p}$ is defined as [20]:

$$
\begin{aligned}
& A_{z}(\tau)=\left.A_{z}\right|_{x(t)=x_{p}}(\tau)=E\left\{\left.z(t+\tau)\right|_{x(t)=x_{p}}\right\}= \\
& =\int_{-\infty}^{\infty} z(t+\tau) p\left(\left.z(t+\tau)\right|_{x(t)=x_{p}}\right) d z
\end{aligned}
$$

where $p\left(\left.z(t+\tau)\right|_{x(t)=x_{p}}\right)$ represents the conditional probability density of the signal $z(t)$ for the condition $x=x_{p}$; $E(\cdot)$ is the expected value; $x_{p}$ is the set threshold value. 
In the case of independence of signal $x(t)$ and disturbance $n(t)$, the expected value of the delayed signal $z(t)$ is also independent of $n(t)$ :

$$
\begin{aligned}
& E\left[\left.z(t+\tau)\right|_{X(t)=x_{p}}\right]=E\left[\left.z(\tau)\right|_{X_{p}}\right]= \\
& =E\left[\left.y(\tau)\right|_{x_{p}}\right]+E\left[\left.n(\tau)\right|_{X_{p}}\right]=E\left[\left.y(\tau)\right|_{x_{p}}\right]
\end{aligned}
$$

If the signals $x(t)$ and $y(t)$ are normal together, with average values of zero, then the conditional probability density of the signal $y(t+\tau)$ at the condition $x(t)=x_{p}$, is expressed as follows [19]:

$$
\begin{aligned}
& p\left[\left.y(\tau)\right|_{x_{p}}\right]=\frac{1}{\sigma_{y} \sqrt{2 \pi} \sqrt{1-\rho_{x y}^{2}(\tau)}} \cdot \\
& \cdot \exp \left\{-\frac{2}{2\left[1-\rho_{x y}^{2}(\tau)\right]}\left[\frac{\rho_{x y}(\tau)}{\sigma_{x}} x_{p}-\frac{y(\tau)}{\sigma_{y}}\right]^{2}\right\}
\end{aligned}
$$

with the conditional expected value:

$$
E\left[\left.y(t+\tau)\right|_{x(t)=x_{p}}\right]=E\left[\left.y(\tau)\right|_{x_{p}}\right]=\frac{\sigma_{y}}{\sigma_{x}} \rho_{x y}(\tau) \cdot x_{p}
$$

and conditional variance:

$$
\sigma^{2}\left[\left.y(t+\tau)\right|_{x(t)=x_{p}}\right]=\sigma_{y}^{2}\left[1-\rho_{x y}^{2}(\tau)\right]
$$

The relationship between $A_{z}(\tau)$ and the normalized CCF $\rho_{x z}(\tau)$ is given by the formula:

$$
A_{z}(\tau)=\frac{\sigma_{z}}{\sigma_{x}} \rho_{x z}(\tau) \cdot x_{p}
$$

A good estimate of the conditional expected value is the arithmetical conditional average value of the signal. Its designation in practice consists in detecting mutually uncorrelated moments of transition of the signal $x(t)$ through the level $x_{p}$, run at these moments registering fragments of the delayed signal $z(t)$ and averaging those fragments in the set:

$$
\hat{A}_{z}(l)=\left.\overline{z(l)}\right|_{x(n)=x_{p}}=\left.\frac{1}{M} \sum_{n=1}^{M} z(n+l)\right|_{x(n)=x_{p}}
$$

where $M$ is the number of averaged uncorrelated segments of the delayed signal $z(t)$.

The position of the main maximum of CAV function defines the transmission time delay as in the case of CCF (Fig.3.). a)

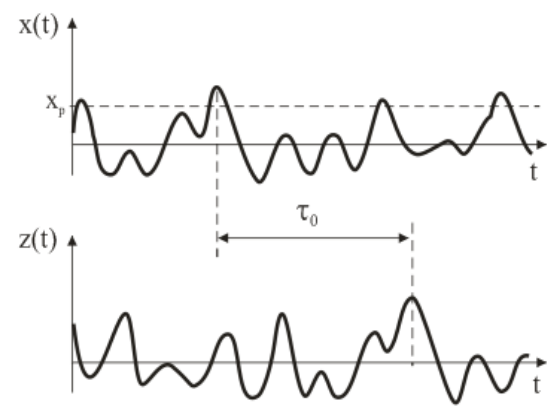

b)

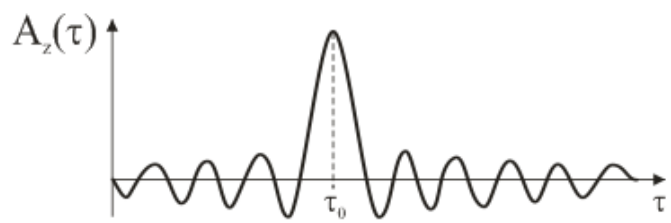

Fig.3. The concept of TDE using CAV function: a) the signals $x(t)$ and $z(t)$, b) the CAV function $A_{z}(\tau)$.

The CAV variance for $M$ averaging is determined by the formula:

$$
\sigma^{2}\left[\hat{A}_{z}(\tau)\right]=\frac{\sigma_{z}^{2}\left[1-\rho_{x z}^{2}(\tau)\right]}{M}
$$

4.1.1. Comparison of standard uncertainties of estimation of CAV and CCF function values in the neighborhood of extreme points

The relative standard uncertainty of the CAV function estimation for $\tau=\tau_{0}$ taking into account (28), (30), (16), and (8) can be presented by [20]:

$$
u_{r e l}\left[\hat{A}_{z}\left(\tau_{0}\right)\right]=\frac{\sigma\left[\hat{A}_{z}\left(\tau_{0}\right)\right]}{A_{z}\left(\tau_{0}\right)}=\frac{1}{\sqrt{M}} \frac{\sigma_{n}}{c x_{p}}=\frac{1}{c \alpha \sqrt{M \cdot S N R}}
$$

where $\alpha=\left(x_{p} / \sigma_{x}\right)$ - relative threshold value.

After dividing equation (31) by (19), the quotient of relative uncertainties of CAV and CCF is obtained as:

$$
\frac{u_{r e l}\left[\hat{A}_{z}\left(\tau_{0}\right)\right]}{u_{r e l}\left[\hat{R}_{x z}\left(\tau_{0}\right)\right]}=\frac{1}{\alpha}\left[\frac{M}{N}\left(2 c^{2} S N R+1\right)\right]^{-1 / 2}
$$

Since CAV and CCF are determined on the basis of uncorrelated signal samples, the $M / N$ quotient may be 1 or less than unity. The plot of the relation $u_{r e l}\left[\hat{A}_{z}\left(\tau_{0}\right)\right] / u_{r e l}\left[\hat{R}_{x z}\left(\tau_{0}\right)\right]=f(S N R)$ for $M / N=1, c=1$ and several selected values of $\alpha$ is shown in Fig.4. In this case, the relative standard uncertainty $u_{r e l}\left[\hat{A}_{z}\left(\tau_{0}\right)\right]$ will be less than $u_{r e l}\left[\hat{R}_{x z}\left(\tau_{0}\right)\right]$ for the value $\alpha \geq 1$. 


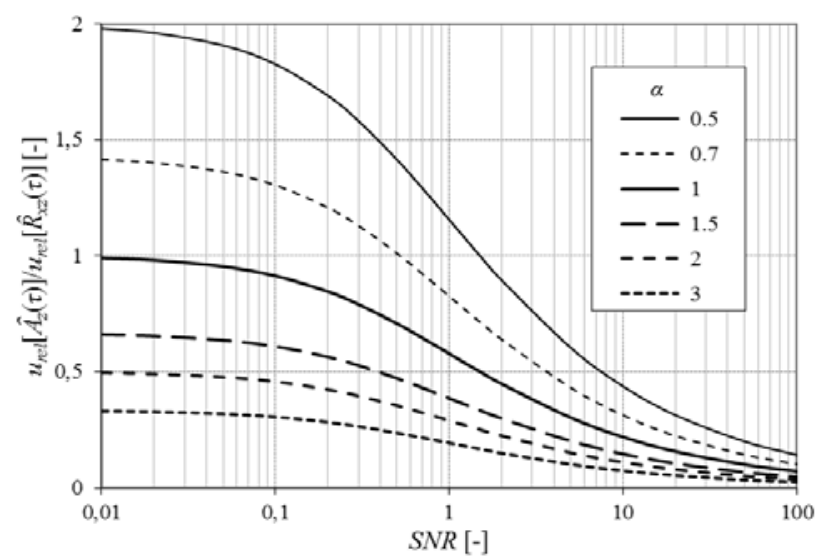

Fig.4. Plot of the relation $u_{r e l}\left[\hat{A}_{z}\left(\tau_{0}\right)\right] / u_{r e l}\left[\hat{R}_{x z}\left(\tau_{0}\right)\right]=f(S N R)$ for $M / N=1, c=1$ and selected values of $\alpha$.

In the work [19] it has been shown that the optimum value of $\alpha$ is about 2. Fig.5. shows a plot of the dependency $u_{r e l}\left[\hat{A}_{z}\left(\tau_{0}\right)\right] / u_{r e l}\left[\hat{R}_{x z}\left(\tau_{0}\right)\right]=f(S N R)$ for $\alpha=2, c=1$ and several selected $M / N$ quotient values.

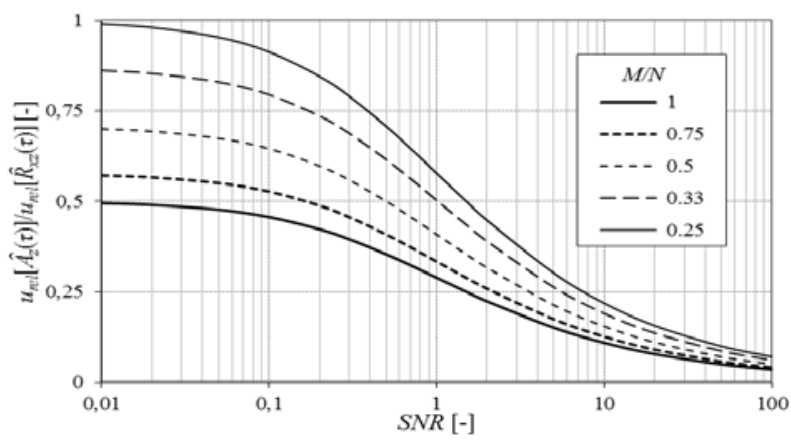

Fig.5. Plot of the relation $u_{r e l}\left[\hat{A}_{z}\left(\tau_{0}\right)\right] / u_{r e l}\left[\hat{R}_{x z}\left(\tau_{0}\right)\right]=f(\operatorname{SNR})$ for $\alpha=2, c=1$ and selected values of $M / N$ ratio.

4.1.2. Comparison of the standard uncertainties of TDE for $C A V$ and $C C F$

For the CAV function, the standard uncertainty of the TDE can be represented by the equation [20]:

$$
u\left[\tau_{0}\right]_{C A V}=\frac{1}{\pi B} \sqrt[4]{\frac{3}{4}} \sqrt{u_{r e l}\left[\hat{A}_{z}\left(\tau_{0}\right)\right]}
$$

After taking into account (30) we get:

$$
u\left[\tau_{0}\right]_{C A V}=\frac{1}{\pi B} \sqrt[4]{\frac{3}{4}} \frac{1}{\sqrt{c \alpha}}(M \cdot S N R)^{-1 / 4}
$$

By comparing (34) and (22) we finally obtain:

$$
\frac{u\left[\tau_{0}\right]_{C A V}}{u\left[\tau_{0}\right]_{C C F}}=\frac{1}{\sqrt{\alpha}}\left[\frac{M}{N}\left(2 c^{2} S N R+1\right)\right]^{-1 / 4}
$$

The plot of the relation $u\left[\tau_{0}\right]_{C A V} / u\left[\tau_{0}\right]_{C C F}=f(S N R)$ for $M / N=1, c=1$ and a few selected values of $\alpha$ is shown in Fig.6.

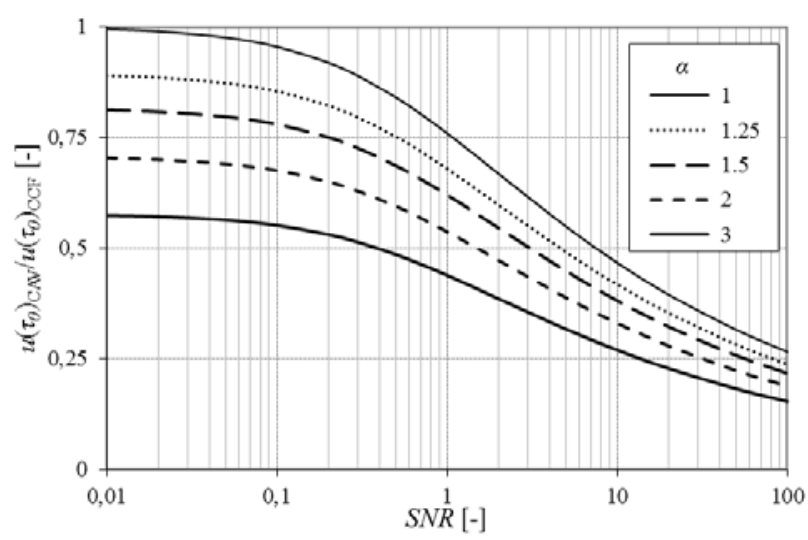

Fig.6. Plot of the relation $u\left[\tau_{0}\right]_{C A V} / u\left[\tau_{0}\right]_{C C F}=f(S N R)$ for $M / N=1, c=1$ and a few selected values of $\alpha$.

Fig.7. shows the plot of the relation (35) for $\alpha=2, c=1$ and a few selected $M / N$ quotient values.

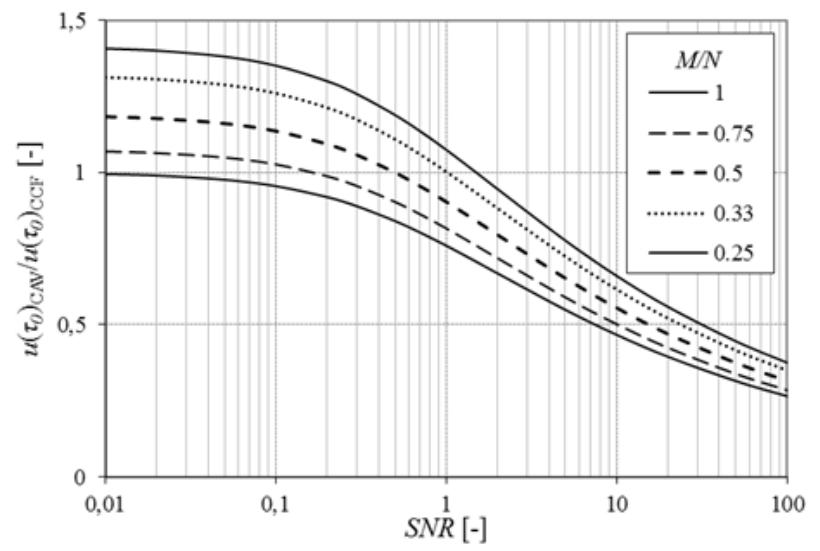

Fig.7. Plot of the relation $u\left[\tau_{0}\right]_{C A V} / u\left[\tau_{0}\right]_{C C F}=f(S N R)$ for $\alpha=2$, $c=1$ and selected values of $M / N$.

Based on Fig.7., it can be stated that the standard uncertainty of TDE using CAV is lower than for CCF independent of SNR values for $\alpha \geq 2$ and $M / N \geq 0.25$.

4.2. Conditional average value of the absolute value of delayed signal

The conditional expected value of the delayed signal absolute value $|z(t)|$ for the condition $x(t)=0$ is defined by the equation [21]:

$$
\begin{aligned}
& A_{|z|}(\tau)=\left.A_{|z|}\right|_{x(t)=0}(\tau)=E\left\{|z(t+\tau)|_{x(t)=0}\right\}= \\
& =\int_{0}^{\infty}|z(t+\tau)| p\left(\left.|z(t+\tau)|\right|_{x(t)=0}\right) d z
\end{aligned}
$$


where $p\left(\mid z(t+\tau) \|_{x(t)=0}\right)$ represents the conditional probability density of the $z(t)$ signal absolute value for the condition $x(t)=0$.

For a normal probability density function $p\left(\mid z(t+\tau) \|_{x(t)=0}\right)$ on the basis of (36), the CAAV $A_{|z|}(\tau)$ is obtained in the form [23]:

$$
A_{|z|}(\tau)=\sigma_{z} \sqrt{\frac{2}{\pi}\left[1-\rho_{x z}^{2}(\tau)\right]}
$$

Transmission time delay is determined by the position of the main minimum of the CAAV function:

$$
\tau_{0}=\arg \left\{\min A_{|z|}(\tau)\right\}=\arg \left\{A_{|z|}\left(\tau_{0}\right)\right\}
$$

After normalization, the equation (37) takes the form:

$$
a_{|z|}(\tau)=\frac{A_{z \mid}(\tau)}{A_{z \mid}(\tau)_{\max }}=\sqrt{1-\rho_{x z}^{2}(\tau)}
$$

Fig.8. shows a comparison of the normalized CAAV $a_{|z|}(\tau)$ and CCF $\rho_{x z}(\tau)$ functions for SNR $=\infty(n(t)=0)$, and $\mathrm{SNR}=5$.

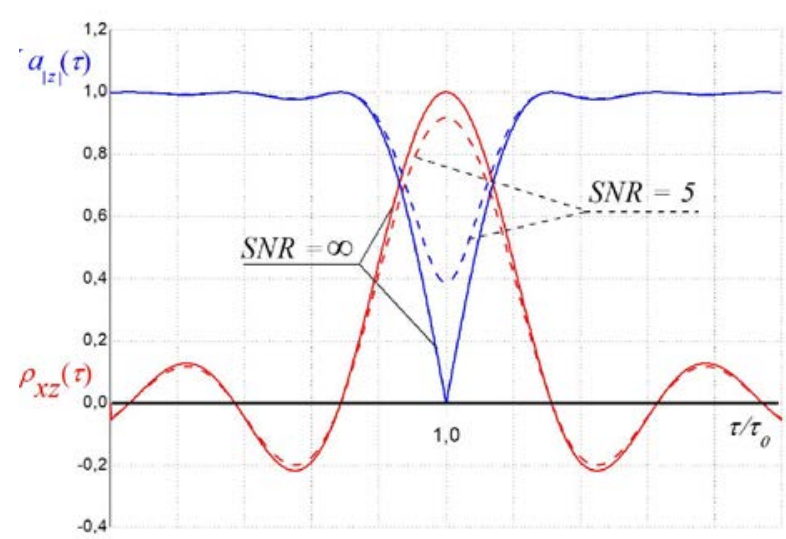

Fig.8. Examples of normalized CAAV $a_{|z|}(\tau)$ and CCF $\rho_{x z}(\tau)$ functions.

The function $a_{|z|}(\tau)$ is characterized by a sharper extremum than the function $\rho_{x z}(\tau)$. A decrease of the SNR value results in a decrease of the main CCF maximum by formula (16) and, respectively, growth of the main CAAV minimum according to:

$$
a_{|z|}\left(\tau_{0}\right)=\sqrt{1-\left(1+\frac{1}{c^{2} S N R}\right)^{-1}}
$$

A good estimate of the expected conditional value (36) is the arithmetical conditional average value of the delayed signal absolute value. Determining the CAAV estimator involves detecting mutually uncorrelated transition times of the original signal $x(t)$ across zero, triggering at these moments registration of fragments of the delayed signal absolute value $|z(t)|$ and averaging those fragments in the set according to the formula:

$$
\hat{A}_{z \mid}(l)=\left.\overline{|z(l)|}\right|_{X(n)=0}=\left.\frac{1}{M} \sum_{n=1}^{M}|z(n+l)|\right|_{X(n)=0}
$$

The variance of the CAAV estimator for $M$ averages is determined by the equation [18]:

$$
\sigma^{2}\left[\hat{A}_{|z|}\left(\tau_{0}\right)\right] \approx \frac{1}{M} \sigma_{z}^{2}\left(1-\frac{2}{\pi}\right)\left[1-\rho_{x z}^{2}\left(\tau_{0}\right)\right]
$$

4.2.1. Comparison of the standard uncertainties of estimation of CAAV and CCF values in the neighborhood of extreme points

Based on equation (42) after taking into account (16), the relative standard uncertainty of the CAAV value estimation is obtained as:

$$
u_{\text {rel }}\left[\hat{A}_{|z|}\left(\tau_{0}\right)\right]=\frac{\sigma\left[\hat{A}_{|z|}\left(\tau_{0}\right)\right]}{A_{|z|}(\tau)_{\max }} \approx\left[\frac{1}{M}\left(\frac{\pi}{2}-1\right) \frac{1}{1+c^{2} S N R}\right]^{1 / 2}
$$

By comparing (43) and (19) we find [23]:

$$
\frac{u_{r e l}\left[\hat{A}_{|z|}\left(\tau_{0}\right)\right]}{u_{r e l}\left[\hat{R}_{x z}\left(\tau_{0}\right)\right]} \approx\left[\frac{N}{M} \frac{\left(\frac{\pi}{2}-1\right)\left(\frac{1}{1+c^{2} S N R}\right)}{\left(2+\frac{1}{c^{2} S N R}\right)}\right]^{1 / 2}
$$

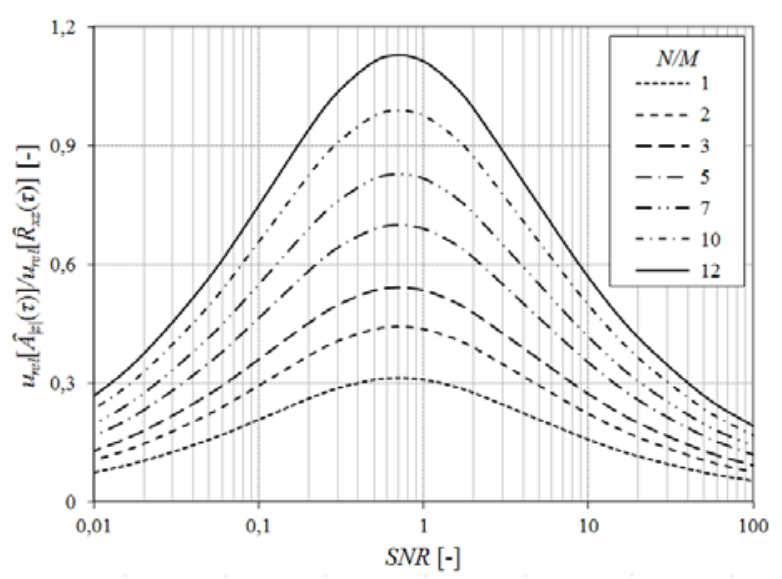

Fig.9. Plots of the relation $\left.u_{r e l} \mid \hat{A}_{|z|}\left(\tau_{0}\right)\right] / u_{r e l}\left[\hat{R}_{x z}\left(\tau_{0}\right)\right]=f(S N R)$ for a few values of $N / M$ ratio [23].

The plot of the relation (44) for $c=1$ and several $N / M$ quotient values is shown in Fig.9. As can be seen in the SNR range under consideration, the relative standard uncertainty of CAAV is always less than the corresponding 
CCF uncertainty for $N / M$ values less than or equal to 10 . In practice, the value of the $N / M$ quotient depends on the interval of the correlation of the measurement signals, which determines the choice of uncorrelated samples.

\subsubsection{Comparison of the standard uncertainties of TDE for CAAV and CCF}

The standard uncertainty of the $\tau_{0}$ transmission time delay estimation from the CAAV function is given by [22]:

$$
u\left[\hat{\tau}_{0}\right]_{C A A V}=\frac{1-\rho_{x z}^{2}\left(\tau_{0}\right)}{\rho_{x z}\left(\tau_{0}\right)}\left[\frac{1}{M}\left(\frac{\pi}{2}-1\right)\right]^{1 / 2}
$$

After taking into account equations (45), (16), and (19), we obtain the relation $u\left[\hat{\tau}_{0}\right]_{C A A V} / u\left[\hat{\tau}_{0}\right]_{C C F}=f(S N R)$ :

$$
\frac{u\left[\hat{\tau}_{0}\right]_{C A A V}}{u\left[\hat{\tau}_{0}\right]_{C C F}}=\left\{\frac{N\left(\frac{\pi}{2}-1\right)}{M c^{2} S N R\left(2 c^{2} S N R+1\right)}\right\}^{1 / 2}
$$

a)

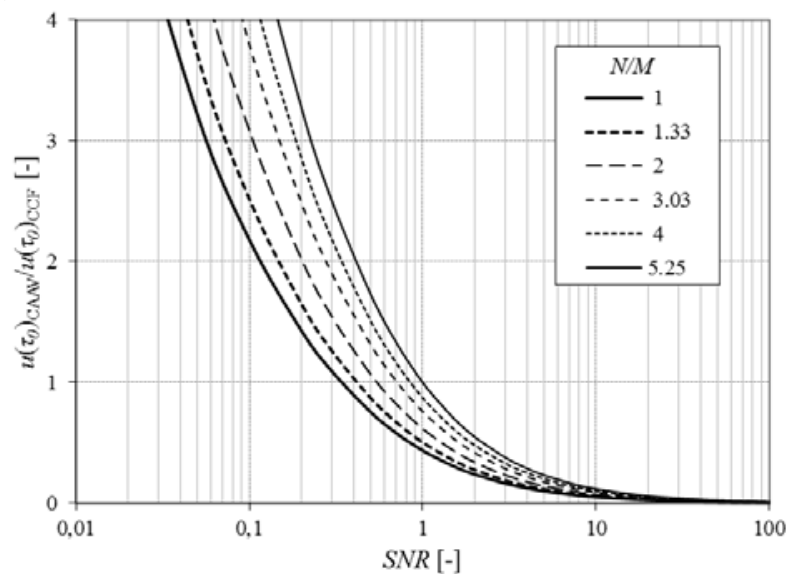

b)

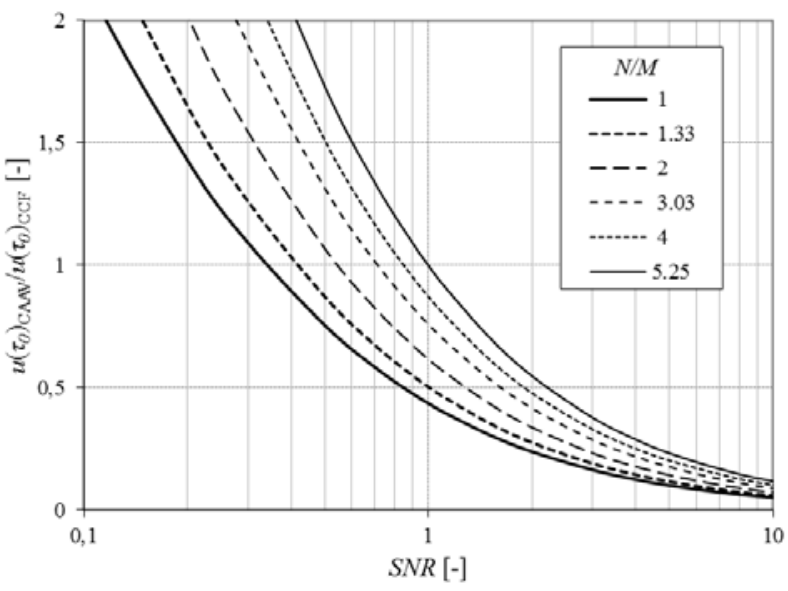

Fig.10. Plots of the relation $u\left[\hat{\tau}_{0}\right]_{C A A V} / u\left[\hat{\tau}_{0}\right]_{C C F}=f(S N R)$ for $c=1$ and a few $N / M$ quotient values; a) SNR range from 0.01 to 100 , b) SNR range from 0.1 to 10 .
The plot of the relation (46) for $c=1$ and several $N / M$ quotient values is shown in Fig.10. Fig.10.b) shows an enlarged area for which the quotient $u\left[\hat{\tau}_{0}\right]_{C A A V} / u\left[\hat{\tau}_{0}\right]_{C C F}$ assumes values close to 1 . Based on the graphs shown in Fig.10, it can be concluded that the standard uncertainty of TDE using CAAV will be less than for CCF for SNR values greater than 0.35 (for $N / M=1$ ). An increase in the $N / M$ quotient results in an increase in the SNR limit value, for which the standard uncertainty of the CAAV time delay estimation is less than for CCF. For SNR $=1$ equalization of the values $u\left[\hat{\tau}_{0}\right]_{C A A V}$ and $u\left[\hat{\tau}_{0}\right]_{C C F}$ occurs for $N / M=5.25$.

\section{SUMMARY}

The article presents the application of two methods of conditional signal averaging to determine the transmission time delay of stochastic signals. These methods are based on analysis of the CAV and CAAV functions. In the first part of the paper we give the models of measurement signals used in the TDE of random signals and, for comparative purposes, the principle and main metrological characteristics of the cross-correlation method.

The main part of the paper presents the concept of application of the conditional averaging to TDE. For discrete CAV and CAAV estimators, the standard uncertainties of the estimation of function values in the neighborhood of the extremes and the standard uncertainties of the TDE were analyzed and compared with the corresponding uncertainties for the direct discrete CCF estimator. For the most commonly used TDE models of measurement signals, the range of SNR values is defined, for which the methods under consideration have lower standard uncertainties of estimation at specified analysis parameters. It was found that the standard uncertainty of TDE using CAV is lower than for CCF independent of SNR values for parameter values of $\alpha \geq 2$ and $M / N \geq 0.25$. The standard uncertainty of TDE applying CAAV will be lower than for CCF for SNR values greater than $0.35(N / M=1)$. An increase in $N / M$ results in an increase in the $S N R$, for which the standard uncertainty of the CAAV time delay estimation is lower than for CCF.

This paper presents the basics and the results of theoretical analysis of CAV and CAAV methods. The authors carried out a simulation and experimental study of the properties of conditional averaging methods in TDE of stochastic signals [23]-[25]. Successful attempts have also been made to use the conditional signal averaging methods in radioisotope measurements of two-phase flows [26], [27].

\section{REFERENCES}

[1] Bendat, J.S., Piersol, A.G. (2010). Random Data: Analysis and Measurement Procedures (Fourth Edition). Wiley.

[2] Assous, S., Linnett, L. (2012). High resolution time delay estimation using sliding discrete Fourier transform. Digital Signal Processing, 22, 820-827. 
[3] Waschburger, R., Kawakami, R., Galvao, H. (2013). Time delay estimation in discrete-time state-space models. Signal Processing, 93, 904-912.

[4] Jacovitti, G., Scarano, G. (1993). Discrete time technique for time delay estimation. IEEE Transactions on Signal Processing, 41 (2), 525-533.

[5] Chen, J., Benesty, J., Huang, Y. (2006). Time delay estimation in room acoustic environments: An overview. EURASIP Journal on Advances in Signal Processing, 2006, 026503.

[6] Hanus, R., Zych, M., Petryka, L., Świsulski, D. (2014). Time delay estimation in two-phase flow investigation using the $\gamma$-ray attenuation technique. Mathematical Problems in Engineering, 2014, 475735.

[7] Chen, J., Huang, Y., Benesty, J. (2004). Time delay estimation. In Audio Signal Processing for NextGeneration Multimedia Communication Systems. Springer, 197-227.

[8] Blok, E. (2002). Classification and evaluation of discrete subsample time delay estimation algorithms. In $14^{\text {th }}$ International Conference on Microwaves, Radar and Wireless Communications (MIKON-2002), May 20-22, 2002. IEEE, 764-767.

[9] So, H.C. (2001). On time delay estimation using an FIR filter. Signal Processing, 81, 1777-1782.

[10] Zych, M., Hanus, R., Vlasak, P., Jaszczur, M., Petryka, L. (2017). Radiometric methods in the measurement of particle-laden flows. Powder Technology, 318, 491500.

[11] Mosorov, V. (2008). Flow pattern tracing for mass flow rate measurement in pneumatic conveying using twin plane electrical capacitance tomography. Particle \& Particle Systems Characterization, 25 (3), 259-265.

[12] Zhang, L., Wu, X. (2006). On the application of cross correlation function to subsample discrete time delay estimation. Digital Signal Processing, 16, 682-694.

[13] Beck, M.S., Pląskowski, A. (1987). Cross-Correlation Flowmeters. CRC Press.

[14] Mosorov, V. (2006). Phase spectrum method for time delay estimation using twin-plane electrical capacitance tomography. Electronics Letters, 42 (11), 630-632.

[15] Hanus, R., Zych, M., Petryka, L., Mosorov, V., Hanus, P. (2015). Application of the phase method in radioisotope measurements of the liquid - solid particles flow in the vertical pipeline. EPJ Web of Conferences, 92, 02020.

[16] Hanus, R. (2015). Application of the Hilbert Transform to measurements of liquid-gas flow using gamma ray densitometry. International Journal of Multiphase Flow, 72, 210-217.

[17] Shors, S.M., Sahakian, A.V., Sih, H.J., Swiryn, S. (1996). A method for determining high-resolution activation time delays in unipolar cardiac mapping. IEEE Transactions on Biomedical Engineering, 43 (12), 1192-1196.
[18] Hanus, R. (2001). Accuracy comparison of some statistic methods of time delay measurements. Systems Analysis Modelling Simulation, 40 (2), 239-244.

[19] Kowalczyk, A., Szlachta, A. (2010). The application of conditional averaging of signals to obtain the transportation delay. Przegląd Elektrotechniczny, 86 (1), 225-228. (in Polish)

[20] Kowalczyk, A., Hanus, R., Szlachta, A. (2011). Time delay estimation of stochastic signals using conditional averaging. In $8^{\text {th }}$ International Conference on Measurement (Measurement 2011), April 27-30, 2011. Bratislava, Slovakia: Institute of Measurement Science SAS, 32-37.

[21] Kowalczyk, A., Szlachta, A., Hanus, R., Chorzępa, R. (2017). Estimation of conditional expected value for exponentially autocorrelated data. Metrology and Measurement Systems, 24 (1), 69-78.

[22] Hanus, R. (2010). Standard uncertainty comparison of time delay estimation using cross-correlation function and the function of conditional average value of the absolute value of delayed signal. Przeglad Elektrotechniczny, 86 (6), 232-235. (in Polish)

[23] Kowalczyk, A., Hanus, R., Szlachta, A. (2011). Investigation of the statistical method of time delay estimation based on conditional averaging of delayed signal. Metrology and Measurement Systems, 18 (2), 335-342.

[24] Szlachta, A., Hanus, R., Kowalczyk, A. (2011). Virtual instrument for the estimation of the time delay using conditional averaging of random signals. In International Workshop on ADC Modelling, Testing and Data Converter Analysis and Design and IEEE 2011 ADC Forum, June 30 - July 1, 2011. IMEKO, 81-86.

[25] Kowalczyk, A., Hanus, R., Szlachta, A. (2016). Time delay measurement method using conditional averaging of the delayed signal module. Przeglad Elektrotechniczny, 92 (9), 279-282.

[26] Hanus, R., Szlachta, A., Kowalczyk, A., Petryka, L., Zych, M. (2012). Radioisotope measurement of twophase flow in pipeline using conditional averaging of signal. In $16^{\text {th }}$ IEEE Mediterranean Electrotechnical Conference (MELECON 2012), March 25-28, 2012. IEEE, 144-147.

[27] Hanus, R., Zych, M., Kowalczyk, A., Petryka, L. (2015). Velocity measurements of the liquid-gas flow using gamma absorption and modified conditional averaging. EPJ Web of Conferences, 92, 02021.

Received February 02, 2018. Accepted July 16, 2018. 